\title{
Microflares to megaflares: Solar observations and modeling
}

\author{
Lyndsay Fletcher \\ School of Physics and Astronomy, SUPA, University of Glasgow, \\ Glasgow G12 8QQ, United Kingdom \\ email: lyndsay.fletcher@glasgow.ac.uk
}

\begin{abstract}
The observationally determined properties of solar flares such as overall energy budget and distribution in space, time and energy of flare radiation, have improved enormously over the last cycle. This has enabled precision diagnostics of flare plasmas and nonthermal particles in large and small events, informing and driving new theoretical models. The theoretical challenges in understanding flare are considerable, involving MHD and kinetic processes operating in an environment far from equilibrium. New observations have also provided some challenges to longstanding models of flare energy release and transport. This talk overviewed recent observational and theoretical developments, and highlighted some important questions for the future
\end{abstract}

Keywords. Sun: flares, activity

Overview Solar flares, defined as rapid (few minutes), localised, transient increases in the solar radiation, are the biggest energy releases in the solar system. The energy release results from the reconfiguration of the coronal magnetic field, most likely by magnetic reconnection. There is a reasonably concensus on the 'big picture' of a flare. Non-linear force free magnetic field reconstructions suggest pre-flare energy is stored in stressed fields less than $10,00 \mathrm{~km}$ above the photosphere, and strongly concentrated around the magnetic neutral line (Sun et al. 2012). The majority of the energy is released and converted, in the few minutes of the flare impulsive phase, into heat, mass motion, and the kinetic energy of non-thermal particles which go on to produce radiation across the electromagnetic spectrum (Emslie et al. 2012). Most - but not all - large flares have an associated CME, and the peak CME acceleration occurs on average about a minute before the peak of the impulsive phase hard X-ray (HXR) energy release (BerkebileStoiser et al. 2012). The bulk of the flare radiation is emitted in the UV and optical part of the spectrum, which is a common flare feature (Kretzschmar 2011) though very poorly observed. Fletcher et al. (2011) gives an up-to-date overview of solar flare observations. Relationship to the evolving magnetic field Chromospheric flare emission is usually ordered into two ribbons of emission, separating in time. It is well known that these ribbons associate well with the calculated locations of the photospheric projections of magnetic quasi-separatrix layers, indicating the importance of the magnetic topology and its evolution. The product of ribbon velocity with the strength of the underlying magnetic field gives a measure of the magnetic reconnection rate. Peaks in this are found to be wellcorrelated with HXR peaks (Qiu 2009), emitted by a few compact HXR sources. The reasons for the location of the HXR sources within the ribbons is not clear; it may reflect the evolution of 'slip-running' reconnection within a QSL (Masson et al. 2009), or it may be linked to particular topological features called separators (Des Jardins et al. 2009). Footpoint properties The flare footpoints - i.e. the HXR and optical emission regions, are the source of most of the impulsive-phase radiant energy. There have not been any UV measurements of these since the 1980s, and optical spectroscopy - except in strong 
lines such as $\mathrm{H} \alpha$ - is almost entirely lacking. So we do not have a clear view of footpoint temperature and density structure, where the energy density is highest. With EUV spectroscopy the picture is better. The Extreme ultraviolet Imaging Spectrometer (EIS) on Hinode has been used for footpoint density and emission measure diagnostics (Graham et al. 2011), flow speed (also available from the Coronal Diagnostic Spectrometer on SOHO) and non-thermal line broadening measurements. The overall picture is that 'coronal' temperature (up to $10 \mathrm{MK}$ ) plasma can also be found in flare footpoints, with densities up to $10^{11} \mathrm{~cm}^{-3}$. 'Sun-as-a-star' observations with SDO/EVE help constrain the continuum (Milligan et al. 2012) but resolved UV and optical observations are a priority Electrons in flares An extensive review by Zharkova et al. (2011) describes the many theoretical models for flare electron acceleration. Flare HXR is usually interpreted in terms of a beam of electrons arriving from the corona, stopping collisionally in the chromosphere and emitting bremsstrahlung. With imaging spectroscopy and WL imaging, very firm lower estimates of the rate of electron arrival per unit area are made, and values up to $10^{20}$ electrons $\mathrm{cm}^{-2} \mathrm{~s}^{-1}$ determined Krucker et al. (2011). For $\sim 30 \mathrm{keV}$ electrons this implies a beam density of $10^{10}$ electrons $\mathrm{cm}^{-3}$. This presents a challenge, as such a beam should be too dense to propagate stably with its return-current. Significant effort is being expended on simulations of beam-return current propagation in the corona. It appears that a large fraction - perhaps $75 \%$ - of the beam energy is dissipated as plasma heating, but the beam continues at the thermal speed, and some energy may even go to electron re-acceleration(Lee \& Büchner 2011; Karlický \& Kontar 2012).

Flares through the cycle Flares are a way for the Sun to shed free energy, and are linked to helicity loss via CME eruptions. At least down to GOES A1 level, flares occur entirely within active regions, based on RHESSI imaging observations (Christe et al. 2008). The flare distribution is roughly a power-law, but the slope of the distribution depends on whether an assessment of thermal or non-thermal energy is made, and the proxy used for the thermal energy. Non-thermal signatures are probably a more robust measure of flare energy, though they do suffer from an unknown low-energy cutoff. The amplitude of the distribution of flare energies varies through the cycle, but its slope does not (Hannah et al. 2011). The flare rate per day is greater at maximum than at minimum. At the end cycle 23 the flare rate per active region was also anomalously high Hudson et al. (2012).

\section{References}

Berkebile-Stoiser, S., Veronig, A. M. , Bein, B. M., \& Temmer, M. 2012, ApJ 753, 88

Christe, S., Hannah, I. G., Krucker, S., McTiernan, J., \& Lin, R. P. 2008, ApJ 677, 1385

Des Jardins, A. \& Canfield, R., Longcope et al. 2009, ApJ 693, 1628

Emslie, A. G. \& Dennis, B. R., Shih A., et al. 2012, ApJ 759, 71

Fletcher, L., Dennis, B. R., Hudson, H. S., et al. 2011, Space Sci. Revs. 159, 19

Graham, D. R., Fletcher, L., \& Hannah, I. G. 2011, A\& $A$ 532, A27

Hannah, I. G., Hudson, H. S., Battaglia, M., et al. 2011, Space Sci. Revs. 159, 263

Hudson, H. S., Fletcher, L., \& McTiernan, J. 2012, Solar Phys. submitted

Karlický, M. \& Kontar, E. P. 2012, A\& A 544, A148

Kretzschmar, M. 2011, A\&A 530, A84

Krucker, S., Hudson, H. S., Jeffrey, N. L. S., et al. 2011, ApJ 739, 96

Lee, K. W. \& Büchner, J. 2011, A $\& A$ 535, A61

Masson, S., Pariat, E., Aulanier, G., \& Schrijver, C. J. 2009, ApJ 700, 559

Milligan, R. O., Chamberlin, P. C., Hudson, H. S., et al. 2012, ApJL 748, L14

Qiu, J. 2009, ApJ 692, 1110

Sun, Z., Hoeksema, J. T. Liu, Y., et al. 2012, ApJ 748, 77

Zharkova, V. V., Arzner, K., Benz, A. O., et al. 2011, Space Sci. Revs. 159, 357 